\title{
Estudio de un compuesto cementante \\ fotoluminiscente y su aplicación en la construcción
}

\section{A study of a photoluminescence cementitious composite and its applications in construction}

Flor Margarita

Ph.D. en Ingeniería

Docente Universidad Central del Ecuador

e-mail: mvflor@uce.edu.ec

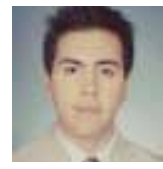

\section{Carpio Andrés}

Egresado de la Carrera de Ingeniería Civil

Universidad Central del Ecuador

e-mail: vacarpio@uce.edu.ec

\author{
Santamaría Jorge. \\ Ph.D. en Ingeniería \\ Docente Universidad Central del Ecuador \\ e-mail: jsantamaria@uce.edu.ec
}

\section{Resumen}

Hoy en día, la necesidad de desarrollar materiales que fomenten el ahorro de energía eléctrica de manera eficiente ha sido uno de los temas trascendentales estudiados alrededor del mundo. Con esta primicia, la presente investigación determinó que la inclusión de Aluminato de Estroncio co-dopado con Europio y Disprosio en mezclas a base de Cemento Portland; no solo produce el fenómeno de fotoluminiscencia, sino que mejora también sus propiedades mecánicas, mediante la realización de diversas pruebas de laboratorio físico-mecánicas como la resistencia a compresión en probetas, además de análisis químicos con XRF en distintas muestras de mortero. La investigación encontró además que la proporciones 0.3:1 del compuesto con respecto al cemento permite obtener el efecto fosforescente más óptimo. Finalmente, mediante la realización de un análisis económico se demuestra que el costo/beneficio de aplicar el compuesto cementante fotoluminiscente como señalética vial a largo plazo es mucho más rentable que el empleo de la luminaria convencional.

Palabras clave: materiales de construcción; cemento; aluminato de estroncio; fotoluminiscencia; fosforescencia, propiedades mecánicas

\section{Abstract}

Nowadays, the need to develop materials that promote efficient energy savings has been one of the transcendental topics studied around the world. With this novelty, the present investigation determined that the inclusion of Strontium Aluminate co-doped with Europium and Dysprosium in binders based on Portland cement not only produces the photoluminescence phenomenon but also improves its mechanical properties; through the accomplishment of diverse physical-mechanical laboratory tests like the compressive strength in test pieces, besides chemical analyzes with XRF in different samples of concrete. The research further found that the 0.3:1 ratio of the compound to the cement allow obtaining the most optimal phosphorescent effect. Finally, by means of the economic analysis it is shown that the cost/ benefit of applying the photoluminescent cementitious composite as a long-term road sign is much more cost effective than the use of the conventional luminaire.

Keywords: construction materials; cement; strontium aluminate; photoluminescence; phosphorescence mechanical properties 


\section{Introducción}

Desde los inicios de la humanidad, esta se ha visto fascinada por los fenómenos de fotoluminiscencia en seres vivos u objetos. Las primeras observaciones sobre este fenómeno datan desde el 1500 A.C. hasta la actualidad, sin embargo, los primeros estudios para lograr su entendimiento no se dieron sino hasta el año 1603 D.C. cuando Vincenzo Cascariolo de Bolonia reportó por primera vez la luminiscencia de los sólidos mediante un experimento en el cual calentó polvos de barita natural (sulfato de bario) con carbón y encontró que la mezcla resultante en forma de torta brillaba en la noche. Él observó que la piedra aparentemente se "cargaba" de luz solar por el día y brillaba durante horas en la oscuridad. Por esto le llamó lapis solaris (piedra del Sol) (Henríquez, s.f.). Conforme ha avanzado el tiempo, se ha ido profundizado no solamente en el estudio de la parte experimental sino, también en el entendimiento teórico del fenómeno de fotoluminiscencia, cuya base fundamental fue descrita en los artículos publicados por Albert Einstein sobre el Efecto Fotoeléctrico en 1905 (Einstein, 1905).

En el campo de la construcción e ingeniería civil, la curiosidad por el empleo de materiales fotoluminiscentes en las obras civiles se manifestó en primer lugar debido a los requerimientos de eficiencia con la señalética de seguridad y posteriormente debido a requerimientos estéticos en la parte arquitectónica con las obras de acabado; lo cual puede constatarse en la obtención de patentes de este tipo (Santolaya, 2014). No obstante su empleo cada vez en crecimiento en las obras civiles ha demandado que se realicen estudios más detallados los cuales tienen como fin, entender el efecto de añadir el compuesto de aluminato de estroncio co-dopado con Eu,Dy $\left(\mathrm{SrAl}_{2} \mathrm{O}_{4}: \mathrm{M}\right.$, donde $\mathrm{M}=\mathrm{Eu}^{2+}$, $\mathrm{Dy}^{3+}$.) en mezclas a base de Cemento Portland Ordinario (CPO) ( Velazco et al. 2014) .

Cabe mencionar que la elaboración y aplicación del compuesto cementante fotoluminiscente (CCF) en el campo de la ingeniería civil, se encuentra protegido por una patente internacional perteneciente a la empresa ITALCEMENTI S.P.A. (Alfani y Lezzi, 2016), por lo cual se aclara que para el presente artículo las cantidades de material elaborado y empleado tuvieron como finalidad simplemente realizar su estudio a nivel académico y sin fines de lucro.

El artículo tiene como finalidad profundizar en el entendimiento del comportamiento físico y de las propiedades mecánicas de los compuestos cementantes fotoluminiscentes; así como también, analizar la influencia del $\mathrm{SrAl}_{2} \mathrm{O}_{4}: \mathrm{M}$ como parte de mezclas basadas en CPO a nivel macroscópico para su aplicación en la in- geniería civil. Se debe recalcar que el estudio a nivel físico-químico, atómico y molecular ha sido ya realizado en otras investigaciones anteriores (Ptáček et al., 2014; Velazco et al., 2014; Ptacek, 2014).

\section{Metodología}

Previo a detallar los procedimientos y pasos seguidos para realizar la presente investigación, se deben aclarar ciertos conceptos a fin de dejar claro los fundamentos y métodos aplicados en el estudio.

\section{Aluminato de estroncio dopado con iones metálicos de tierras raras}

Es limitada la literatura al respecto del método de fabricación y síntesis del $\mathrm{SrAl}_{2} \mathrm{O}_{4}: \mathrm{M}$ propiamente; sin embargo, de manera breve se puede dar a conocer el cómo se obtiene este compuesto. Para producir en primer lugar el $\mathrm{SrAl}_{2} \mathrm{O}_{4}$ se mezclan íntimamente cantidades equimoleculares de óxido de aluminio y óxido de estroncio con aproximadamente $1 \%$ de ácido bórico, el cual actúa como fundente. Posteriormente se calcinan a $1100^{\circ} \mathrm{C}$ en atmósfera reductora con hidrógeno durante un aproximado de 10 horas. La mezcla es sacada y molida, y nuevamente se la calcina por otras 10 horas; este proceso se repite varias veces hasta lograr el tamaño de partícula deseado. Sin embargo, el efecto fotoluminiscente del $\mathrm{SrAl}_{2} \mathrm{O}_{4}$ no podría ser posible sin la presencia de los activadores o dopantes como se les conoce comúnmente. Para el caso del aluminato de estroncio dichos dopantes añadidos por lo gene-ral corresponden a los iones metálicos de "tierras raras" (Eu+ $\mathrm{Eu}^{2+}, \mathrm{Gb}^{3+}, \mathrm{Dy}^{3+}, \mathrm{Sm}^{3+}, \mathrm{Tb}^{3+}$, etc.) los cuales exhiben una alta eficiencia cuántica y fosforescente (Rojas, 2015; Gschneidner et al. 1999).

De manera particular el $\mathrm{SrAl}_{2} \mathrm{O}_{4}: \mathrm{M}$ difiere de otros compuestos fosforescentes similares como el Sulfuro de Zinc codopado con Cobre o Cobalto (ZnS:Cu,Co) por su persistencia lumínica; es decir, el $\mathrm{SrAl}_{2} \mathrm{O}_{4}: \mathrm{M}$ presenta una persistencia lumínica hasta 10 veces más que la de los compuestos de sulfuro dopados (Rojas, 2015; Inan Akmehmet et al, 2016).

\section{El cemento}

Debido al fin que tiene ésta investigación y a que en el Ecuador no se produce propiamente CPO sino sus variantes con adiciones (Tipos IP o GU), se decidió emplear un Cemento Portland Blanco Tipo I (CPO-B-I) de importación y producido por la empresa CEMEX (México). De las pruebas de laboratorio realizados al mismo se obtuvieron los principales valores que inciden en el cálculo de la dosificación del CCF: Densidad absoluta del cemento $\delta a b=3.18 \mathrm{gr} / \mathrm{cm} 3$ (ASTM International, 2016a), Densidad aparente del cemento 
$\delta a p=1.30 \mathrm{gr} / \mathrm{cm} 3$, y la cantidad de agua que se requiere para lograr la consistencia normal de la pasta agua.cn\%=26.36 \% (ASTM International, 2016b).

\section{Agregado fino}

Como parte del CCF y con la finalidad de abaratar su costo mediante la inclusión de partículas de mayor tamaño y que ocupan mayor volumen, se realizó la inclusión de agregado fino (arena) la cual cumple con las respectivas normativas técnicas y requisitos de calidad a nivel nacional (Normas INEN) e internacional (Normas ASTM) para fabricación de morteros, siendo usada arena procedente del sector de Santo Domingo de los Tsáchilas de la mina Copeto, Ecuador, con pro-piedades como su Módulo de Finura MF=2.47 (ASTM International, 2016c), Peso específico $\gamma=2.37 \mathrm{~g} / \mathrm{cm}^{3}$ y Capacidad de absorción Abs\%=3.07 \% (ASTM International, 2015a), sin presencia de impurezas orgánicas, figura 1 (transparente) (ASTM International, 2016d) y su respectiva curva y límites granulométricos (ASTM International, 2014).

\section{La fotoluminiscencia y período de persistencia}

Rojas (2015) en su trabajo de investigación realizó un análisis minucioso desde un trasfondo físico-químico sobre este punto, además de detallar la parte experimental para la medición de la persistencia. No obstante, en la presente investigación se abordarán estos conceptos de manera general y práctica.

La fotoluminiscencia de manera general se produce cuando un compuesto codopado con ciertos elementos es sometido a una excitación mediante una fuente lumínica por un cierto período de tiempo o exposición, es decir, se carga el compuesto; y una vez suspendida o apagada la fuente de excitación, el compuesto empieza a emitir luz. En el caso del $\mathrm{SrAl}_{2} \mathrm{O}_{4}: \mathrm{M}$ la fotoluminiscencia se debe a que durante el proceso de absorción de energía los electrones de los iones metálicos saltan de las órbitas internas a las externas, y cuando "caen" de nuevo a su estado original emiten un fotón de luz. Dicha luz y su color dependen del elemento activador y la longitud de onda $(\lambda)$ que emite este, así pues, tomando como ejemplo el ZnS:M, éste emite en azul ( $\lambda=452 \mathrm{~nm})$ si se dopa con $\mathrm{Eu}^{3+}$, en ver-de $(\lambda=510 \mathrm{~nm})$ si se incorpora con $\mathrm{Cu}^{2+}$ y $\mathrm{Co}^{2+}$ o en amarillo $(\lambda=570 \mathrm{~nm})$ al doparlo con $\mathrm{Mn}^{2+} \mathrm{y} \mathrm{Ce}^{2+}$.

Por otra parte, la persistencia de la luminiscencia se puede definir como la energía almacenada en el compuesto debido a fenómenos de "trapping" (captura) de los elementos activadores una vez han sido excitados, es decir, que alcanza estado metaestable del sistema cuántico que tiene un nivel de energía superior a estado fundamental. El cuan prolongada sea la lumi- niscencia dependerá de lo lenta que sea la liberación de carga de los activadores.

\section{El método de dosificación y mezcla del CCF}

De forma general, no existe un método establecido para dosificar exactamente un compuesto cementante o mortero (CC), la mayoría de códigos, normativas o investigaciones basan su dosificación en tablas empíricas (Satyarno, 2014; Grijalva y Laines, 2016; INEN, 2014). Para la presente investigación se propuso innovar a fin de poder obtener una base comparable al momento de analizar los resultados, y esto se lo logró realizando un diseño de CC teniendo como objetivo el obtener la mayor resistencia a compresión ( $\left.f^{\prime} c\right)$ posible en los especímenes. Esto es posible si se considera trabajar con la relación agua/cemento (a/c) mínima posible concerniente al porcentaje necesario para alcanzar una consistencia normal en la pasta de cemento, descartando de momento el efecto que tiene el agregado dentro de toda la matriz cementante.

Evidentemente la pregunta que surge de momento es: ¿Por qué trabajar con un mortero y no con hormigón?, pues la respuesta recae en el factor económico al momento en que se añade el compuesto fotoluminiscente al mortero. En el caso ecuatoriano el costo aproximado de un $1 \mathrm{~kg}$ de $\mathrm{SrAl}_{2} \mathrm{O}_{4}: \mathrm{M}$ es de

US\$260.00; no obstante la interrogante que ahora debería formularse es: ¿Cuál es el objetivo y fin del CCF?, dado el alto costo que tiene producir $1 \mathrm{~m} 3$ del CCF ya que lo que interesa es la emisión de luz a nivel superficial, lo más factible y el uso previsto del CCF es como capa de revestimiento o sustituto de señales plásticas fotoluminiscentes; por lo cual el desarrollar una mezcla de hormigón fotoluminiscente no tendría sentido, y por lo tanto lo más factible resulta desarrollar un mortero de recubrimiento.

Las proporciones de las mezclas de CCF y CC se las puede observar en la Tabla I. Como se puede apreciar en las muestras M1, M2, M3, las dosificaciones indicadas no contemplan las correcciones por absorción ni humedad de la arena por motivos prácticos, no obstante, las mismas si fueron consideradas al momento de la realización de las mezclas. Por otro lado, de las pruebas de fluidez realizadas a los morteros (ASTM International, 2015b) se obtuvieron los siguientes valores para las muestras M1, M2, M3, P2 y P3 respectivamente: $\mathrm{FM} 1=106 \%, \mathrm{FM} 2=107 \%, \mathrm{~F}_{\mathrm{M} 3}=109 \%, \mathrm{~F}_{\mathrm{P} 2}$ $=105 \%$ y $F_{P 3}=106 \%$, lo cual indica que cumplen con el rango recomendado de $110 \pm 5 \%$ para el tipo de prueba considerada. 


\begin{tabular}{c|r|r|r|r}
\hline \multirow{2}{*}{$\begin{array}{c}\text { MUESTRA/ } \\
\text { DOSIFICACIÓN }\end{array}$} & \multicolumn{5}{|c}{ MATERIAL } \\
\cline { 2 - 5 } & AGUA (W) & CEMENTO (C) & ARENA (A) & $\mathrm{SrAl}_{2} \mathrm{O}_{4}$ (AS) \\
\hline M1 & 0.27 & 1.00 & 3.00 & 0.30 \\
\hline M2 & 0.27 & 1.00 & 2.00 & 0.30 \\
\hline M3 & 0.27 & 1.00 & 1.00 & 0.30 \\
\hline P2 & 0.27 & 1.00 & 2.00 & 0.00 \\
\hline P3 & 0.27 & 1.00 & 1.00 & 0.00 \\
\hline
\end{tabular}

Además, se puede ver que los morteros tienen una inclusión de $\mathrm{SrAl}_{2} \mathrm{O}_{4}: \mathrm{M}$ de $30 \%$ con respecto a la masa de cemento empleada. Dicha proporción fue adoptada teniendo en cuenta los resultados obtenidos por Grijalva, et. al. (2016), mismos que demostraron que la inclusión de ese porcentaje de $\mathrm{SrAl}_{2} \mathrm{O}_{4}: \mathrm{M}$ en el mortero logra la máxima eficiencia en la luminiscencia (Grijalva y Laines, 2016). Las mezclas patrón son las correspondientes a P2 y P3; sin embargo, debe mencionarse que no se realizó una mezcla patrón para M1 ya que será demostrado más adelante que ésta es la menos efectiva en resistencia y luminiscencia, por lo que fue descartada.

Finalmente, para terminar con la explicación de la parte experimental se estableció un programa de pruebas y ensayos de laboratorio con el objetivo de estudiar el comportamiento físico-mecánico y químico del CCF, quedando determinado el realizar pruebas de: Compresión Simple en Cubos de Mortero, Pruebas de Persistencia de Fotoluminiscencia y Fluorescencia de Rayos X (XRF del inglés X Ray Fluorescence). La cantidad total de probetas elaboradas fue de 90 cubos, distribuidos en 15 cubos por cada muestra los cuales fueron ensayados en grupos de 5 por cada día.

\section{Resultados y discusión}

Los resultados obtenidos en las pruebas de compresión realizadas a los diferentes tipos de muestras ensayadas a edades de 3, 7 y 28 días, pueden apreciarse en la Figura 1. Particularmente se debe destacar la interacción observada entre la matriz compuesta por el CPO-B-I y el $\mathrm{SrAl}_{2} \mathrm{O}_{4}: \mathrm{M}$ con el agregado fino, donde a simple vista era apreciable que en edades tempranas de 3 y 7 días la propagación de las grietas y fracturas en los especímenes se daban en la zona de transición de la interface (ZTI) de la matriz cementícia de CPO-B-I y el $\mathrm{SrAl}_{2} \mathrm{O}_{4}: \mathrm{M}$ al evidenciarse desprendimientos entre la pasta y el agregado. Además, la ausencia de un corte o fisuras en los agregados que se encontraban en el plano de propagación de las grietas (ver Fig. 2). No obstante, a la edad de 28 días, en todas las muestras, fue evidenciable ya el trabajo en conjunto entre la matriz cementícia y el agregado.

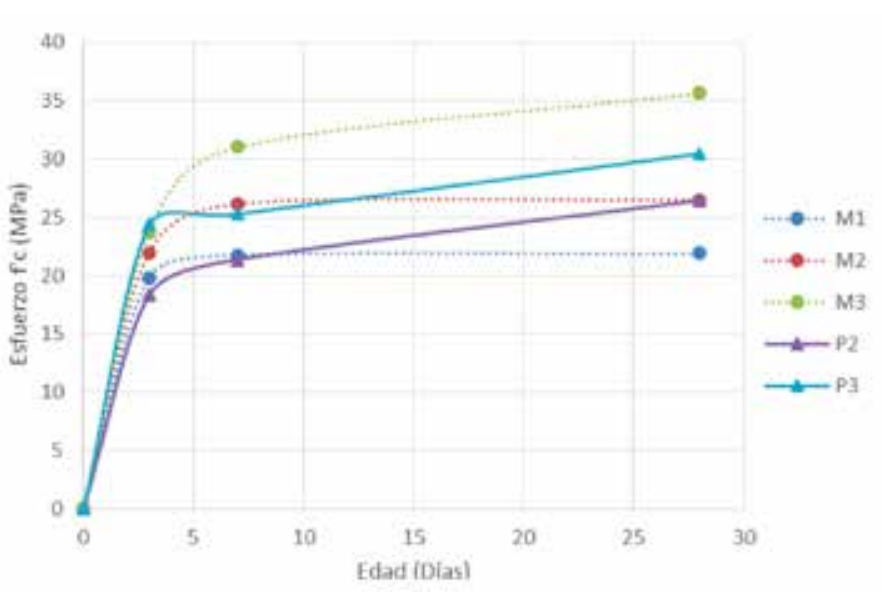

Figura. 1. Curvas "Edad-Resistencia" CCF y CC.

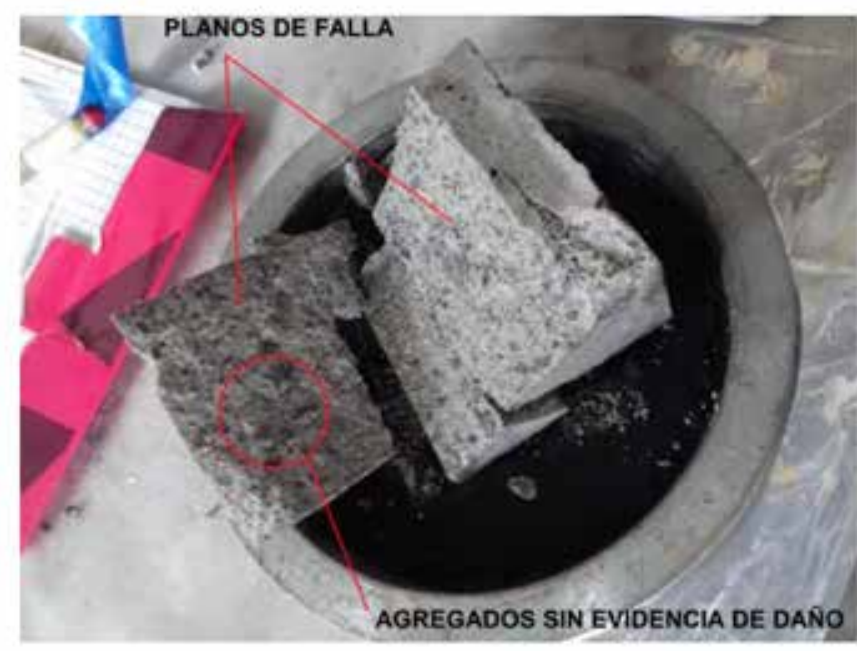

Figura. 2. Fotografía de uno de los especímenes de M2 de 3 días de edad, se aprecia el modo de propagación de la grieta y la textura de la falla.

Por otro lado, se puede observar además la evolución en la resistencia a compresión respecto a su edad de las diferentes muestras. La muestra M2, aún cuando presenta resistencias elevadas a edades tempranas, a los 28 días llega a ser inferior a la de la muestra patrón. Se debe mencionar también que no se realizó una muestra patrón para la muestra M1 debido a que de pruebas piloto iniciales se llegó a observar que era la muestra que tenía menos intensidad lumínica y por tanto se descartó su análisis.

Referente a lo peculiar de la curva de la muestra P3 observado en la Figura 1, este tipo de comportamiento ha sido también apreciable en las investigaciones de Carpio (2016) y Grijalva, et. al. (2016) donde se trabaja de igual manera con CPO-B-I (Carpio, 2016.; Grijalva y Laines, 2016). Luego de revisar la respectiva literatura y realizar un análisis minucioso, se puede determinar que este comportamiento es debido en primer lugar a la composición química del cemento Portland con el cual se está trabajando, ya que como es bien conocido, los CPO-B-I son ricos en Silicato Dicálcico (C2S), lo que produce altas resistencias a edades tardías, pero 
su contenido de Aluminatos (C3A y C4AF) es baja, lo cual influencia en la resistencia a edades tempranas. En segundo lugar, se debe también a la distribución granulométrica de todo el compuesto; sin embargo, aún cuando la evolución de resistencia desde el día 0 al 3 y del 7 al 28 es sostenida y progresiva; la interrupción apreciable entre el día 3 y 7 es debido en mayor parte a las fases de hidratación que tiene el cemento, como es manifestado en obras específicas que estudian la química del cemento (Newman y Choo, 2003; Labahn y Kohlhaas, 1985). Se debe destacar además que en las mezclas M1, M2 y M3 se debió realizar correcciones por la absorción de agua del $\mathrm{SrAl}_{2} \mathrm{O}_{4}: M$ ya que en ensayos previos realizados a este compuesto se determinó que es capaz de absorber aproximadamente el 25\% de su peso en agua, lo cual de no tomarse en cuenta puede alterar los resultados.

En la Fig. 3 se puede observar de forma comparativa los resultados de las diferentes muestras respecto a su edad y tipo de muestra. De estos resultados se puede establecer que las mezclas con proporciones M3 presentan un mejor desempeño físico- mecánico y que la inclusión del $\mathrm{SrAl}_{2} \mathrm{O}_{4}: \mathrm{M}$ aporta a la mejora del CC.

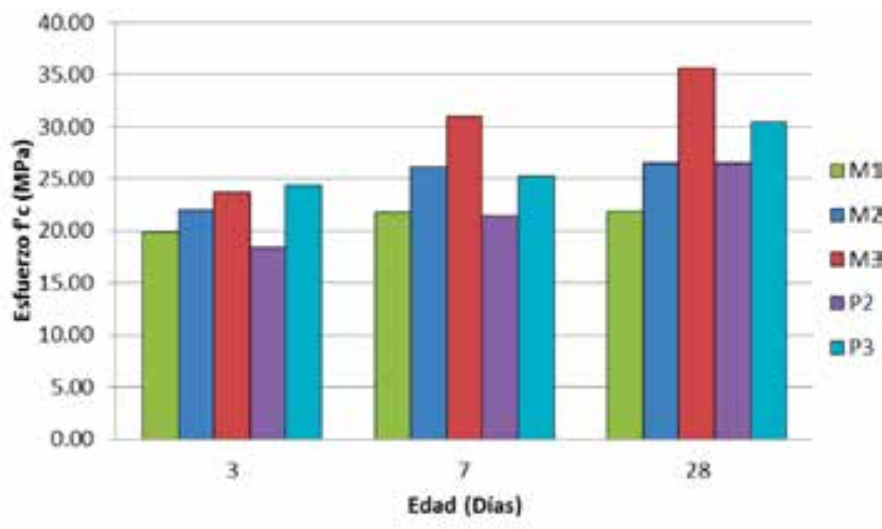

Figura. 3. Gráfica comparativa de Resistencias a Compresión vs. Edad.

La Tabla 2 muestra los resultados procedentes de las pruebas de Fluorescencia de Rayos $X$ realizadas a una muestra de CPO-B-I hidratada con agua en una relación $\mathrm{a} / \mathrm{C}=0.27$ en proporciones de $\mathrm{P} 3$ y a otra muestra de $\mathrm{CPO}-\mathrm{B}-\mathrm{I}+\mathrm{SrAl}_{2} \mathrm{O}_{4}: \mathrm{M}$ en proporciones de M3 hidratada de igual manera con una relación al $\mathrm{c}=0.27$ más la respectiva cantidad de agua por corrección para el $\mathrm{SrAl}_{2} \mathrm{O}_{4}: \mathrm{M}$. La prueba para ambas muestras fue realizada a la edad de 28 días. Se tomó como base de estudio la mezcla tipo M3 debido a que fue la que mejores propiedades mecánicas y lumínicas presentó. Analizando el comportamiento químico se puede establecer claramente la incidencia del $\mathrm{SrAl}_{2} \mathrm{O}_{4}: \mathrm{M}$ reaccionando con el CPO-B-I y como resultado se tiene la disminución en los Óxidos de Silicio así como el claro incremento en Óxidos de Aluminio y Óxidos de Estroncio, lo cual hace que el CCF tenga propiedades refractarias (como se pue- de observar con la disminución en las pérdidas por calcinación). Por otra parte, se evidencia también el incremento en los Óxidos de Fósforo; sin embargo, no se superan los límites permisibles por lo cual no representan un peligro. También se observa la disminución en los Óxidos de Magnesio y con lo que se puede evitar la posible formación de Hidróxido de Magnesio $\mathrm{Mg}(\mathrm{OH})_{2}$. Mismo que al expandirse puede llegar a provocar grietas y deteriorar el CCF.

\begin{tabular}{|c|c|c|}
\hline Muestra & \multicolumn{2}{|c|}{$\begin{array}{c}\text { Pérdidas por } \\
\text { calcinación (\%) }\end{array}$} \\
\hline Cemento & & 23.7 \\
\hline \multicolumn{2}{|c|}{ Cemento $+\mathrm{SrAl}_{2} \mathrm{O}_{4}$} & 6.07 \\
\hline Elemento & $\begin{array}{c}\text { Cemento } \\
\text { Contenido (\%) }\end{array}$ & $\begin{array}{c}\text { Cemento + } \\
\mathrm{SrAl}_{2} \mathrm{O}_{4}(\%)\end{array}$ \\
\hline $\mathrm{SiO}_{2}$ & 13.18 & 12.50 \\
\hline $\mathrm{Al}_{2} \mathrm{O}_{3}$ & 2.75 & 18.70 \\
\hline $\mathrm{Fe}_{2} \mathrm{O}_{3}$ & 0.16 & 0.22 \\
\hline $\mathrm{CaO}$ & 54.77 & 48.73 \\
\hline $\mathrm{K}_{2} \mathrm{O}$ & 0.12 & 0.11 \\
\hline $\mathrm{TiO}_{2}$ & 0.05 & 0.05 \\
\hline $\mathrm{Na}_{2} \mathrm{O}$ & $<0.01$ & $<0.01$ \\
\hline $\mathrm{MgO}$ & 0.75 & 0.59 \\
\hline $\mathrm{P}_{2} \mathrm{O}_{5}$ & 0.05 & 0.28 \\
\hline $\mathrm{SO}_{3}$ & 2.81 & 0.83 \\
\hline $\mathrm{SrO}$ & - & 18.20 \\
\hline $\mathrm{ZrO}_{2}$ & - & 0.80 \\
\hline $\mathrm{V}_{2} \mathrm{O}_{5}$ & - & 0.04 \\
\hline $\mathrm{CuO}$ & - & 0.02 \\
\hline $\mathrm{NiO}$ & - & 0.02 \\
\hline $\mathrm{ZnO}$ & - & $<0.01$ \\
\hline
\end{tabular}

Debido a la inclusión del SrAl2O4:M se incorpora a la mezcla Metales Pesados como el Cobre y Zinc los cuales pueden llegar a inhibir el fraguado del cemento; sin embargo, no se evidenció este efecto a nivel macroscópico. Del mismo modo se puede distinguir una clara disminución en los Óxidos de Azufre, así como la aparición de elementos como el Zirconio los cuales fomentan la formación de sulfuros y con esto aportan al efecto de fotoluminiscencia que el CCF muestra.

Para la realización de las pruebas de luminiscencia se tomó como base para su ejecución la guía establecida en la normativa ASTM E2073-10 (ASTM International, 2010) y DIN 67510-1 (DIN, 2009); sin embargo, debido a las limitantes con el equipo de laboratorio disponible se adaptaron las pruebas a fin de llegar a cumplir con lo establecido en las respectivas normativas ya que no se contaba con un equipo capaz de medir la luminiscencia (luminance del inglés) directamente, por lo cual se empleó un equipo MASTECH MS6610 para medir la iluminación (illuminance del inglés); es decir, se realizó la medición de iluminación emanada directamente por una superficie dentro de una determinada área. Las mediciones fueron reportadas en lux, y posteriormente para una mayor precisión y comprensión fueron transformadas a porcentaje a fin de mejorar su interpretación. En 
primer lugar, con la finalidad de conocer si el espesor del CCF aplicado en una determinada área influía directamente en la cantidad de luminiscencia, se elaboraron probetas de $5 \times 15 \mathrm{~cm}$ de área con espesores de 5, 4, 3 y 2 milímetros de las mezclas M1, M2 y M3, dichas muestras fueron ubicadas en orden como se lo puede apreciar en la Fig. 4. Posterior se procedió a realizar la carga lumínica del CCF mediante una exposición a luz solar directa por un tiempo de 8 horas.

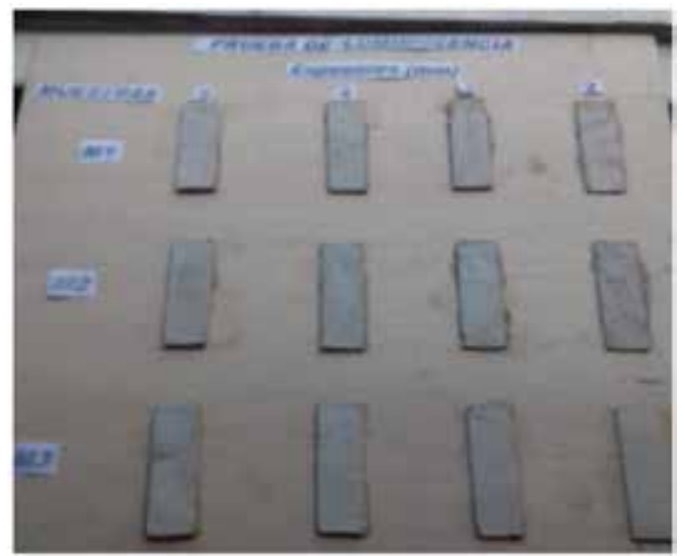

Figura. 4. Prueba de Luminiscencia - Muestra vs. Espesor

En la Fig. 5 se puede observar el proceso de medición de una muestra correspondiente al polvo de SrAl2O4:M, con una superficie de $15 \times 15$ centímetros, dando como medida final un valor de luminiscencia de aproximadamente 36 lux medidos a una distancia de 0.4 metros. Aplicando el factor de conversión a candela (Iv(cd) $=\mathrm{Ev}(\mathrm{Ix}) \times(\mathrm{d}(\mathrm{m})) 2$; donde Ev es la intensidad en lux y d la distancia en metros) se obtuvo una medición de aproximadamente $5.76 \mathrm{~cd} / \mathrm{m} 2$ o $5760 \mathrm{mcd} / \mathrm{m}^{2}$, lo cual coincide con datos obtenidos por investigaciones sobre el material (Hirata et al., 2005). Este valor excede en 25.76 veces el máximo reportado en la normativa ASTM E2073-10; por lo cual, acorde a la adaptación que se ha realizado para la presente investigación, el valor de 36 lux será el dato referencial y equivaldrá al 100\% de intensidad lumínica obtenible en un $\mathrm{T}_{0}=0$ minutos.

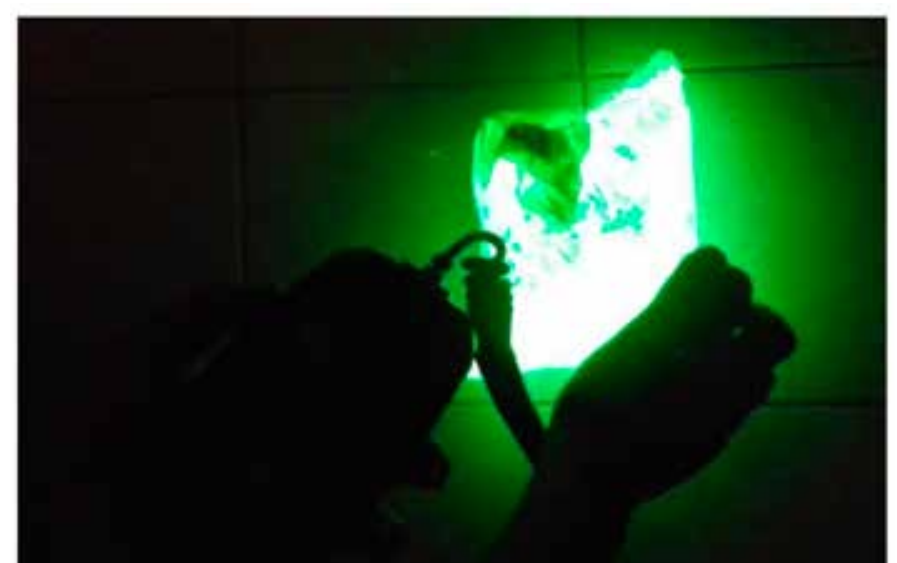

Figura. 5. Medición de la intensidad lumínica del polvo de SrAl204:M colocada a un período de exposición a luz solar "natural" (Tiempo de exposición = 8 horas)

Como se puede apreciar en la Fig. 6, el CCF presenta fosforescencia y la muestra que presenta una mayor intensidad lumínica es la mezcla M3, las mezclas M1 y M2 presentan aproximadamente la misma intensidad lumínica. No obstante, se llegó a observar que la intensidad lumínica manifestada en las muestras de CCF no depende de su espesor sino de la cantidad y concentración del compuesto de CPO-B-I + SrA$\mathrm{I}_{2} \mathrm{O}_{4}: \mathrm{M}$ que conforman el mortero y mezcla como tal. Esto se lo puede constatar observando los valores registrados en la Fig. 7.

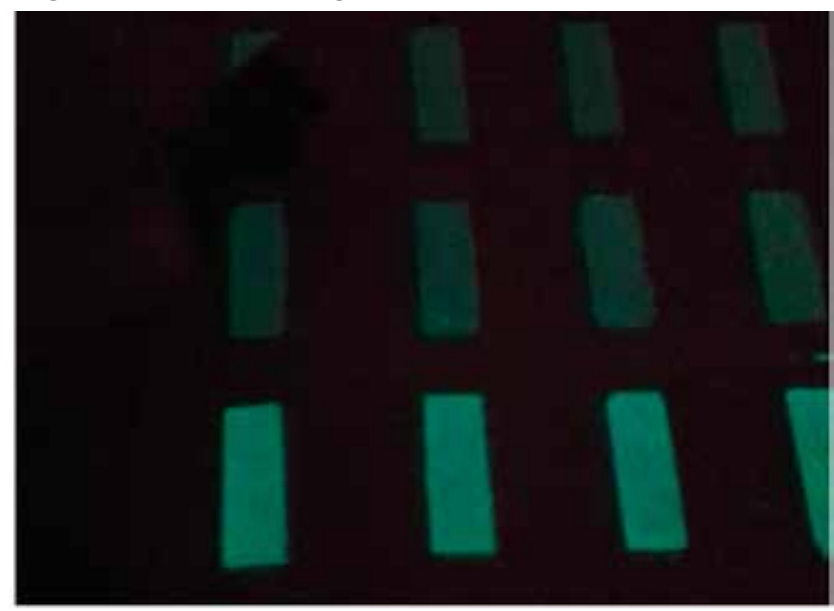

Figura. 6. Período de Emisión de las muestras en ambiente controlado.

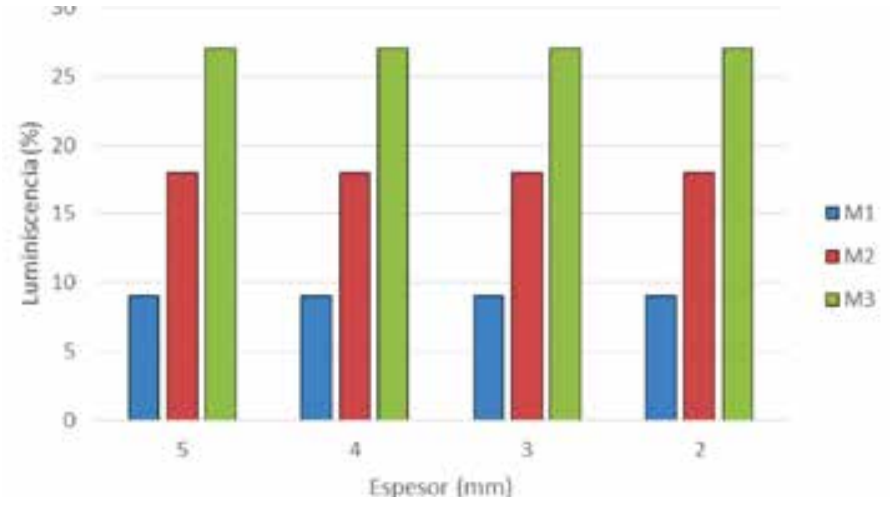

Figura. 7. Gráfica comparativa de las pruebas de luminiscencia para las muestras de CCF M1, M2y M3.

Una vez determinado que la mezcla M3 es la que presenta mayor intensidad lumínica al igual que las mejores propiedades mecánicas, se procedió a realizar la prueba de luminiscencia conforme lo especifica la normativa ASTM E2073-10, en 5 especímenes con dimensiones de $15 \times 15$ centímetros de superficie, donde se obtuvo que el valor de luminiscencia inicial aproximado para éste CCF fue de 10 lux medidos a una distancia de 0.4 metros, lo cual en unidades de candela da un valor de $1.6 \mathrm{~cd} / \mathrm{m} 2$ o $1600 \mathrm{mcd} / \mathrm{m} 2$. Este valor obtenido de 10 lux será el nuevo dato referencial y equivaldrá al $100 \%$ de intensidad lumínica obtenible en un T0 =0 minutos para de esta manera obtener las respectivas curvas de decaimiento.

Los resultados de las pruebas de luminiscencia mostrados en la Tabla 3 son el resultado de un estudio riguroso realizado durante un lapso de 4 meses de manera continua, desde el 1 de mayo de 2017 hasta el 31 de agosto de 2017, habiendo obtenido un total de 5535 registros en los 123 días. Las mediciones de 
intensidad lumínica mostradas en la Tabla 3 fueron determinadas en base al promedio de los valores registrados diariamente para cada intervalo de tiempo indicado también en la tabla por cada mes. Las probetas empleadas para el estudio se encontraban dentro de una habitación con ambiente controlado conforme lo indica en la normativa ASTM E2073-10 durante la realización del ensayo. El procedimiento principalmente difirió con lo normado en lo que respecta al tipo de excitación a la que fue sometido el CCF durante el tiempo carga, mismo que consistió en luz solar por un lapso de 8 horas. Aún cuando existieron días de lluvia o en los que existía poca luminosidad debido al clima, se encontró la particularidad de que durante la etapa de emisión del CCF desde la intensidad inicial hasta la final tenían un comportamiento similar a cuando las probetas eran expuestas a luz solar directa sin interrupción. Esto se debe particularmente a que la mayor parte de la energía que actúa para la excitación del CCF proviene de la radiación UV como lo manifiestan estudios previos (Rojas, 2015; Inan Akmehmet et al.,2016; Ptacek, 2014).

Tabla 3. Resumen de resultados de las pruebas de luminiscencia en probetas de mezcla M3

\begin{tabular}{c|c|c|c|c}
\hline \multirow{2}{*}{ TIEMPO } & \multicolumn{4}{|c}{ INTENSIDAD } \\
\cline { 2 - 5 } & \multicolumn{4}{|c}{$\%$} \\
\hline Minutos & MES 1 & MES 2 & MES 3 & MES 4 \\
\hline 0 & 100 & 100 & 100 & 100 \\
\hline 5 & 90 & 95 & 95 & 90 \\
\hline 10 & 85 & 80 & 85 & 80 \\
\hline 20 & 70 & 75 & 75 & 75 \\
\hline 40 & 60 & 60 & 60 & 60 \\
\hline 60 & 50 & 55 & 50 & 50 \\
\hline 120 & 40 & 40 & 35 & 35 \\
\hline 240 & 20 & 20 & 20 & 20 \\
\hline 480 & 10 & 10 & 10 & 10 \\
\hline & & & &
\end{tabular}

En la Fig. 8 se aprecia claramente la tendencia de las curvas de decaimiento, específicamente en el mes 4 se observa que el CCF tiende a tener un comportamiento lumínico más homogéneo. Entre el minuto 0 al 120, la persistencia del CCF decae rápidamente y del minuto 120 en adelante se observó un decaimiento lento y sostenido.

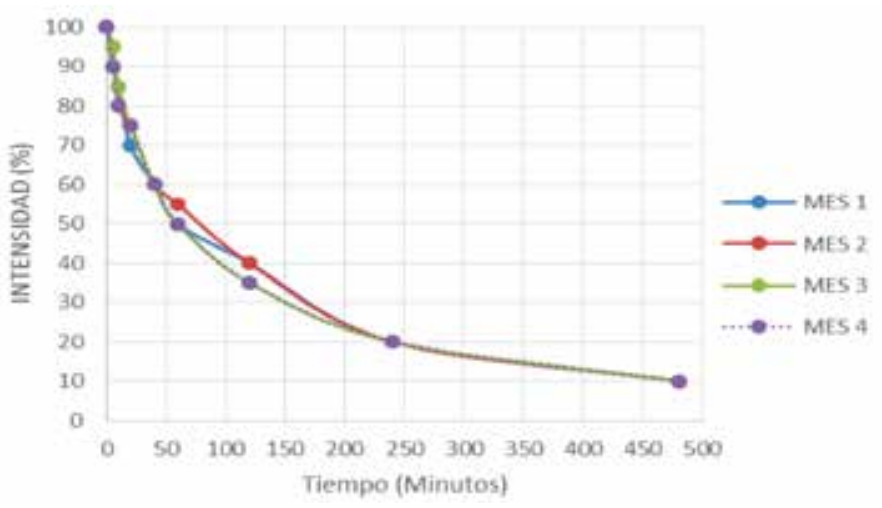

Figura. 8. Curvas de decaimiento obtenidas para probetas de mezclas M3.
Como parte final de esta investigación se realizó un análisis económico referencial comparativo entre las diferentes mezclas de CCF y mezclas patrones CC realizadas; así como el análisis del costo de producción y mantenimiento de la luminaria convencional en las diferentes vías y autopistas dentro del país. Para este análisis económico referencial los precios fueron obtenidos y promediados de fuentes fiables como la Cámara de la Industria de la Construcción de Quito, así como de cotizaciones realizadas directamente a proveedores. En la Tabla 4 y Tabla 5 se puede observar los diferentes costos concernientes a la materia prima, productos finales y mantenimiento sin considerar los costos concernientes a mano de obra e instalación. Para el caso del CCF es evidente que la inclusión del $\mathrm{SrAl}_{2} \mathrm{O}_{4}: \mathrm{M}$ en la mezcla de mortero convencional eleva su costo a niveles altos para el caso de este tipo de CC; sin embargo, debido al fin y utilidad con el que fue concebido su desarrollo principal, es decir para ser empleado como señalética de seguridad o vial, el costo resulta ser competitivo e inclusive se observa un ahorro económico en el largo plazo.

Como se aprecia en caso de la mezcla M3 que fue la escogida debido a sus excelentes propiedades mecánicas y lumínicas, aunque posee un costo más elevado que las lámparas de alumbrado público inicialmente, debido a la vida útil que presenta el CCF (más de 8 años) termina siendo más económico en el largo plazo. Si se tiene en cuenta lo expresado en el RTE INEN 069 que versa sobre alumbrado público y las diferentes condiciones y especificaciones que este debe cumplir, menciona que la separación entre postes de alumbrados debe cumplir con la relación $\mathrm{S} / \mathrm{H}=(3.5$ a 4), dónde $\mathrm{S}$ es la separación entre postes y $\mathrm{H}$ la altura del poste dependiendo de la clase de iluminación empleada; es decir que si tomamos una clase de iluminación "M5" la cual como requisito establece 10 lux de luminancia, por cada metro de altura del poste, se considera que la distancia horizontal de influencia lumínica se irá incrementando en 4 metros. Por lo que tomando estos 4 metros lineales como base de análisis para realizar la comparación y si se considera que de $1 \mathrm{~m} 2$ de CCF se puede obtener 4 metros lineales de $5 \mathrm{~mm}$ de espesor y 250 mm de ancho; la inversión inicial del CCF será de US\$ 366.26, sin embargo, esta es una sola inversión debido a su vida útil. Por otro lado, la inversión inicial de una lámpara de alumbrado público es de US\$237.62, pero dado a que anualmente debe renovarse dicha inversión en 8 años (que es lo que dura el CCF) terminará siendo de US\$1900.96. Por lo tanto, se constata el beneficio de emplearse el CCF como una alternativa para la iluminación vial o de señalética de seguridad en las diferentes obras civiles. 
Actualmente, a modo investigativo y de estudio, las diferentes mezclas de CCF (M1, M2 y M3) han sido aplicadas a la intemperie en una Obra Civil localizada en Machala-Ecuador (ver Fig. 9 y Fig. 10), para evaluar su desempeño y durabilidad en el transcurso del tiempo; no obstante, los resultados de ese análisis permitirán realizar otros artículos en los cuales se detallarán los resultados de esas investigaciones a largo plazo.

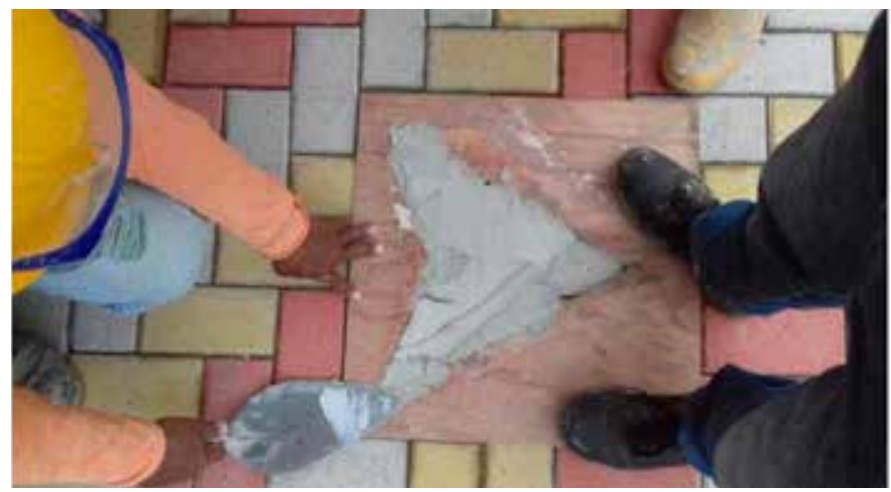

Figura. 9. Colocación de una mezcla M3 como señalética en los exteriores del Poli-deportivo de Machala - Ecuador.

PRODUCTO: MORTERO FOTOLUMINISCENTE DESCRIPCIÓN: $1 \mathrm{~m} 2$-Espesor=5mm VIDAUTIL:+8AÑOS

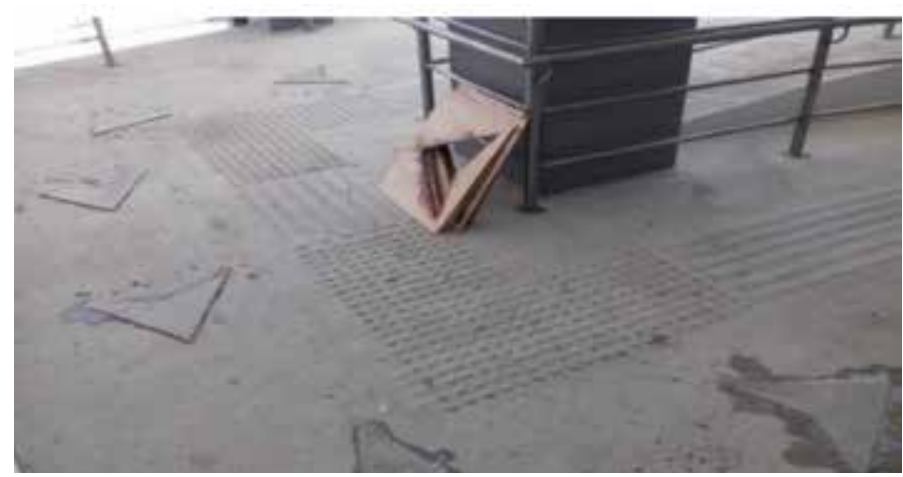

Figura. 10. Señalética de seguridad elaborada con mezcla M2 colocada en las rampas de acceso y emergencia en el Coliseo 3000 de Machala.

\section{Conclusiones}

Las mezclas M1 y M2 presentan resistencias a edades tempranas mayores gracias a la influencia del SrAl2O4. Sin embargo, a los 28 días llegan a ser inferiores a las resistencias de las mezclas patrones, esto debido a la reducción de C2S por las reacciones iniciales entre el SrAl2O4 con el C3A y el C4AF.

La luminiscencia en los CCF no depende del espesor de la muestra sino del área que ocupa y el contenido de $\mathrm{SrAl} 2 \mathrm{O} 4$ en el compuesto, como se pudo evidenciar en la Figura 7 y los parámetros de la expresión matemática que permite el cálculo de la intensidad lumínica. Es decir, que la persistencia e intensidad no dependen del volumen de mezcla ocupado. Desde luego, por la complejidad de colocar en obra espesores menores a $1 \mathrm{~mm}$ y debido también al tamaño de partícula del árido fino, se recomienda no ocupar espesores inferiores a los $2 \mathrm{~mm}$.

En el estudio de persistencia lumínica de la muestra M3 se llega a determinar que a pesar de la duración de la prueba ésta tiende a ser similar y no decae con el pasar del tiempo.

El CCF debe preferentemente colocarse en una superficie sin terminado liso (rugosa), debido a que la difusión del compuesto fotoluminiscente al interior de la mezcla hace que el realizar un terminado liso de la superficie, la intensidad lumínica de los activadores se vea reducida debido a que la matriz cementante recubre las partículas que generan el efecto fotoluminiscente.

El CCF es factible de emplearse como sustituto de la señalética plástica de seguridad y emergencia empleada actualmente en las obras civiles, debido a que al ser el CCF un material cementício con excelentes propiedades refractarias, en el caso de incendios o eventos donde existan altas tempera-

Tabla 4. Análisis económico referencial comparativo de las distintas mezclas de CCF y CC

\begin{tabular}{|c|c|c|c|c|c|c|c|c|c|c|c|c|}
\hline \multirow{2}{*}{ ELEMENTO } & \multirow{2}{*}{ Unidad } & \multirow{2}{*}{ P.U USS } & \multicolumn{2}{|c|}{ M1 } & \multicolumn{2}{|c|}{$\mathrm{M} 2$} & \multicolumn{2}{|c|}{ M3 } & \multicolumn{2}{|c|}{\begin{tabular}{|c|} 
P2 \\
\end{tabular}} & \multicolumn{2}{|c|}{ P3 } \\
\hline & & & Cantidad & Costo USS & Canticlad & Costo USS & Cantidad & Costo USS & Cantidad & Costo USS & Cantichad & Costo USS \\
\hline AGUA (W) & litros & 0.001 & 1.323 & \begin{tabular}{|l|}
0.00 \\
\end{tabular} & 1.699 & \begin{tabular}{|l|}
0.00 \\
\end{tabular} & 2.373 & \begin{tabular}{|l|}
0.00 \\
\end{tabular} & 1.699 & \begin{tabular}{|l|}
0.00 \\
\end{tabular} & \begin{tabular}{|l|}
2.373 \\
\end{tabular} & 0.00 \\
\hline CEMENTO (C) & $\mathrm{Kg}$ & 0.460 & 2.540 & 1.17 & 3.267 & 1.50 & 4.563 & 2.10 & 3.267 & 1.50 & 4.563 & 2.10 \\
\hline ARENA (A) & $\mathrm{Kg}$ & 0.002 & 7.633 & 0.02 & 6.534 & 0.01 & 4.563 & 0.01 & 6.534 & 0.01 & 4.563 & 0.01 \\
\hline $\mathrm{SrAl}_{2} \mathrm{O}_{4}$ (AS) & $\mathrm{Kg}$ & 266 & 0.763 & 202.96 & 0.98 & 260.68 & 1.369 & 364.15 & 0 & 0.00 & 0 & 0.00 \\
\hline & & UBTOTAI & & 204.14 & & 262.20 & & 366.26 & & 1.52 & & 2.11 \\
\hline
\end{tabular}

Tabla 5 . Análisis económico concerniente al costo de producción y mantenimiento de lámparas de alumbrado público

PRODUCTO: LAMPARA PARA POSTE DE LUZ

VIDA UTIL: 1 AÑ̃

\begin{tabular}{c|c|c|c|c}
\cline { 2 - 5 } & Valor USS & Costo (Shora) & C.Consumo (\$año) & C. TOTAL (S/año) \\
\hline Lampara (200W) & 150 & 0.02 & 87.6 & 237.62
\end{tabular}


turas, el CCF perdurará guiando de esta manera a las personas hacia el lugar de encuentro ante una emergencia.

\section{Agradecimientos}

Los autores agradecen a la CONSTRUDIPRO S.A. por el financiamiento de la investigación además de las facilidades brindadas en las diferentes obras de infraestructura en la ciudad Machala - Ecuador. A la empresa ADMIX LTDA., por el apoyo técnico y logístico para la realización de las pruebas de laboratorio.

\section{Referencias bibliográficas}

Alfani, R. y G. Lezzi (2016). Photoluminescent cementitious compositions based on hydraulic binders particularly suitable for use in safety signs, Italcementi S.P.A. (Bergamo, IT), WO 2016062873 A 1.

ASTM International. (2010). ASTM E2073-10, Standard Test Method for Photopic Luminance of Photoluminescent (Phosphorescent) Markings, West Conshohocken, PA, doi: 10.1520/E2073-10

ASTM International. (2014). ASTM C136/C136M - 14: Standard test method for sieve analysis of fine and coarse aggregates, West Conshohocken, PA, doi: 10.1520/C0136_C0136M-14

ASTM International. (2015a). ASTM C128 - 15: Standard test method for relative density (specific gravity) and absorption of fine aggregate, West Conshohocken, PA, doi: 10.1520/C0128-15

ASTM International. (2015b). ASTM C1437-15: Standard Test Method for Flow of Hydraulic Cement Mortar, ASTM International, West Conshohocken, PA, doi: 10.1520/C1437-15

ASTM International. (2016a). ASTM C188 - 16, Standard test method for density of hydraulic cement.", West Conshohocken, PA, doi: 10.1520/ C0188-16

ASTM International. (2016b). ASTM C187-16, Standard Test Method for Amount of Water Required for Normal Consistency of Hydraulic Cement Paste, West Conshohocken, PA, doi: 10.1520/C0187-16

ASTM International. (2016c). ASTM C33/C33M16e1, Standard Specification for Concrete Aggregates, West Conshohocken, PA, doi:10.1520/ C0033_C0033M-16E01

ASTM International. (2016d). ASTM C40/ C40M-16:Standard Test Method for Organic Impurities in empresa doi:10.1520/C0040_ C0040M-16

Carpio, V. (2016). Análisis experimental de un hormigón elaborado con residuos industriales de polvo de grafito. IV Congreso REDU. ESPE, Sangolquí, Ecuador.

DIN. (2009). DIN 67510-1: Phosphorescent pigments and products - Part 1: Measurement and marking at the producer.

Grijalva, F. y Laines, T. (2016). Diseño de morteros fotoluminiscentes aplicados de forma ornamental y señalización en caso de emergencia para edificaciones. (Tesis de Pregrado). Universidad Central del Ecuador, Quito, Ecuador.

Einstein, A. (1905). "Concerning an Heuristic Point of View Toward the Emission and Transformation of Light." Annalen der Physik 17,322(6), pp.132-148.

Henríquez, B. (s.f.). "La luz sin llamas: breve historia de la luminiscencia (primera parte)." Recuperado de:http://www.cubasolar.cu/biblioteca/ energia/En ergia14/HTML/articulo07.htm

Hirata, Y., T. Sakaguchi y N. Takeuchi (2005). Phosphorescence exhibiting phosphor and Fine Aggregates for International, West Concrete, ASTM Conshohocken, PA, Gschneidner, K.A., Eyring, L. y Lander, G.H. eds. (1999). Handbook on the physics and chemistry of rare earths (Vol. 26). Elsevier. process for producing the same, Nemoto \& Co., Ltd., WO2005044944 A1.

INEN. (2014). Código de practica ecuatoriano: CPE INEN-NEC-SE-MP 26-6. Capítulo 6: Mampostería Estructural. Quito, Ecuador.

Rojas, R. (2015). Diseño y Síntesis de Materiales Nanoestructurados basados en Aluminatos de Estroncio con Propiedades Fotoluminiscentes. (Tesis doctoral). Universidad Politécnica de Madrid, España.

Santolaya, P. (2014). Pieza de hormigón luminiscente. E. Tecment tecnologia y gestion constructiva SL. (Valencia, ES), ES1124406U.

Velazco, G., Almanza, J.M., Cortés, D.A., Escobedo, J.C., y Escalante-Garcia., J.I. (2014). Effect of the strontium aluminate and hemihydrate contents on the properties of a calcium sulphoaluminate based cement. Materiales de Construcción, 64 (315), Julio-Septiembre, doi:10.3989/mc.2014.04413 